Claremont Colleges

Scholarship@ Claremont

All HMC Faculty Publications and Research

HMC Faculty Scholarship

2-1-1997

\title{
Photoluminescence Properties of Silicon Quantum-Well Layers
}

Peter N. Saeta

Harvey Mudd College

A. C. Gallagher

University of Colorado at Boulder

\section{Recommended Citation}

"Photoluminescence properties of silicon quantum well layers," P. N. Saeta and A. C. Gallagher, Phys. Rev. B 55, 4563 (1997). doi: 10.1103/PhysRevB.55.4563

This Article is brought to you for free and open access by the HMC Faculty Scholarship at Scholarship @ Claremont. It has been accepted for inclusion in All HMC Faculty Publications and Research by an authorized administrator of Scholarship @ Claremont. For more information, please contact scholarship@cuc.claremont.edu. 


\title{
Photoluminescence properties of silicon quantum-well layers
}

\author{
P. N. Saeta* and A. C. Gallagher \\ JILA, University of Colorado and National Institute of Standards and Technology, Boulder, Colorado 80309-0440
}

(Received 26 August 1996)

\begin{abstract}
Nanometer-scale crystal silicon films surrounded by $\mathrm{SiO}_{2}$ were prepared by oxidizing silicon-on-insulator substrates prepared from SIMOX (separation by implantation of oxygen) and crystallized hydrogenated amorphous silicon films. Average silicon layer thickness was determined from reflection spectra. When sufficiently thin $(<2 \mathrm{~nm})$, all layers emitted red photoluminescence under blue and UV cw excitation, with a spectrum that did not depend on the mean layer thickness. The spectrum was roughly Gaussian with a peak energy of 1.65 $\mathrm{eV}$, which is lower than for most porous silicon spectra. The time scale for the luminescence decay was $\sim 35 \mu$ s at room temperature and $\sim 54 \mu \mathrm{s}$ at $88 \mathrm{~K}$; the decay was nonexponential and did not exhibit spectral diffusion. Atomic force microscope images of the silicon layers showed that luminescing layers were broken apart into regions $\sim 50-100 \mu \mathrm{m}$ in diameter, suggesting that luminescence comes only from regions small enough to have no nonradiative recombination centers in the band gap. These results are inconsistent with a simple quantum-confinement model for luminescence in two-dimensional silicon and suggest the importance of radiation from surface states. [S0163-1829(97)02704-5]
\end{abstract}

\section{INTRODUCTION}

Bulk crystal silicon does not luminesce efficiently because nonradiative recombination processes at defects and impurities proceed much more rapidly than phonon-assisted radiative transitions. Recently there has been great interest in the luminescence properties of nanocrystalline silicon (nc-Si) stimulated by the 1990 discovery of strong room-temperature photoluminescence from anodically etched porous silicon. ${ }^{1}$ Among over 1000 papers that have appeared in the literature since then, the vast majority concern the fabrication, characterization, and behavior of porous silicon prepared by etching crystal silicon $(c-\mathrm{Si})$, either by passing an electric current (anodic etching) or without current (stain etching). A comparative few have focused attention on nanocrystalline silicon materials prepared in other ways. While porous silicon has the great virtue of being easy to prepare, it has many undesirable properties. These include the mechanical and photochemical fragility of the resulting material; its essential inhomogeneity, which makes it difficult to identify the luminescent species, to understand the luminescence mechanism in detail, and to obtain high luminescence efficiency; and the great difficulty in making satisfactory electrical contacts. Since an important technological goal is to create efficient electroluminescent devices out of silicon in ways that are compatible with conventional silicon processing for microcircuit fabrication - thereby opening the way to sophisticated and inexpensive integrated electronics and optoelectronicsthe poor electroluminescence efficiency of porous silicon remains a significant obstacle.

Nanocrystalline silicon materials prepared by alternative methods have a number of advantages. These include the possibility of fabrication in ways more compatible with conventional silicon processing; more careful control of the chemical environment of the Si nanocrystals, including their doping and the composition of the surrounding material; a more homogeneous size and/or shape distribution of the nanocrystalline particles; and a more accurate characteriza- tion of their dimensions. Realization of these possibilities can help clarify the essential physics of nc-Si luminescence and may point the way to efficient electroluminescent devices.

Several techniques for making nc-Si have been explored, including gas-phase production via the thermal decomposition of silanes ${ }^{2,3}$ and microwave plasma decomposition of silane, ${ }^{4} \mathrm{Si}$ deposition in the presence of large partial pressures of hydrogen, ${ }^{5,6}$ spark ablation of $c$-Si surfaces, ${ }^{7}$ laser and thermal crystallization of amorphous silicon/silicon nitride layers, ${ }^{8}$ and Si-rich oxide layers. ${ }^{9,10}$ Studies of the materials produced by these techniques have shown that nc-Si does luminesce strongly in the red on the microsecond time scale, and sometimes blue ${ }^{11}$ on the nanosecond time scale, ${ }^{12}$ when successfully passivated to minimize rapid nonradiative surface recombination. However, they do not unambiguously identify the source of the luminescence, nor are they mutually consistent. For example, while Littau et al. ${ }^{2}$ and Kanemitsu ${ }^{3}$ have prepared oxide-coated Si nanocrystals, they report different luminescence properties of these nominally identical structures. Littau et al. see a blueshift in the luminescence spectrum for distributions of nanocrystals with smaller mean diameter ${ }^{2}$ and steadily increasing intensity with decreasing temperature, ${ }^{13}$ whereas Kanemitsu et al. see no dependence on size and a steep drop in intensity below 100 K. ${ }^{14}$ While some measurements are readily explained by phonon-assisted recombination ${ }^{15}$ in the nanocrystals, others appear more consistent with recombination at localized sites at the surface of the nanocrystal. ${ }^{16}$

An idea central to many theories of nc-Si luminescence is quantum confinement: when the dimension of the Si crystal becomes comparable to or smaller than the free exciton size, the electron and hole wave functions sense the boundaries of the crystal and their energies are shifted further from midgap by heightened localization. The confinement energy shift is greatest for zero-dimensional "dots," but appreciable energy shifts are also predicted for confinement in one-dimensional wires and two-dimensional sheets or layers. ${ }^{17}$ Furthermore, 
confinement tends to concentrate the oscillator strength of the band-edge transition into the exciton peak and to enhance the exciton binding energy. ${ }^{18}$ In indirect materials such as silicon, this might also lead to enhanced oscillator strength by increasing the momentum-space extent of the electron and holes states, and therefore their wave-function overlap. ${ }^{19}$ It is reasonable to suppose, therefore, that confinement effects in thin Si layers may shift the band-edge luminescence to higher energy and may lead to improved radiative efficiency compared to bulk Si.

\section{Thin silicon layers}

Thin silicon layers between insulators have only onedimensional confinement, but offer a number of diagnostic and practical advantages. These include relatively easy construction by layered deposition and a well-characterized thickness. Previous work on Si layers has involved two essentially different approaches. The first is patterned after the success of the GaAs/AlAs system and uses $\mathrm{Ge}$ as the complementary material to produce structures that luminesce in the infrared. $\mathrm{Si}_{x} \mathrm{Ge}_{1-x}$ has a narrower band gap than $c$-Si, so a $\mathrm{Si}_{x} \mathrm{Ge}_{1-x}$ layer between $c$-Si layers forms a quantum well whose band gap can be tuned by varying either the Ge concentration or the well width. ${ }^{20}$ Alternatively, one can take advantage of zone folding to map the silicon conduction-band minimum to the zone center in an epitaxially grown $\mathrm{Si}_{m} \mathrm{Ge}_{n}$ superlattice. ${ }^{21}$ Both approaches have led to improved luminescence at low temperature, but the efficiency drops sharply on heating to room temperature.

The second approach involves crystallizing hydrogenated amorphous silicon $(a-\mathrm{Si}: \mathrm{H})$ layers either with rapid thermal or laser annealing. Chen et $a l^{22}$ grew $a-\mathrm{Si}: \mathrm{H} / a-\mathrm{SiN}_{x}: \mathrm{H}$ multilayers using plasma-enhanced chemical-vapor deposition (PECVD), which they annealed with a cw $\mathrm{Ar}^{+}$laser. Raman scattering and $\mathrm{x}$-ray diffraction were used to show that the typical crystallite size was roughly equal to the layer thickness of $\sim 3.5 \mathrm{~nm}$. After annealing, the multilayer structure showed a broad photoluminescence peak centered at 2.1 $\mathrm{eV}(590 \mathrm{~nm})$ that was not present in the as-deposited material, and which the authors attributed to a one-dimensional confinement effect. Grützmacher et al. ${ }^{8}$ looked at the dependence of the photoluminescence (PL) spectrum on Si layer thickness in the same $a-\mathrm{Si}: \mathrm{H} / a-\mathrm{SiN}_{x}: \mathrm{H}$ multilayer system crystallized with 325 -nm laser light or rapid thermal annealing. They reported a broad PL spectrum extending $\sim 450-700 \mathrm{~nm}$ from the as-deposited multiquantum-well structure, the peak of which narrows, shifts to $\sim 475 \mathrm{~nm}(2.6 \mathrm{eV})$, and grew tenfold in intensity on annealing. They also saw a slight blueshift with decreasing layer thickness in the range $0.6-3.0 \mathrm{~nm}$, suggesting a quantumconfinement mechanism. High-resolution cross-sectional TEM images of laser-crystallized $a-\mathrm{Si}: \mathrm{H} / a-\mathrm{SiN}_{x}: \mathrm{H}$ layers showed that the layers consist of roughly round crystalline particles limited in size by the layer thickness and surrounded by presumably amorphous material. ${ }^{23}$ Thus these systems appear as densely packed quantum dots, not as twodimensional (2D) crystalline layers, and there is reason to suspect that nitrogen, ${ }^{7}$ oxygen,${ }^{24}$ or $\mathrm{OH}$ groups ${ }^{25}$ in the surrounding material are responsible for the luminescence, and not simply quantum confinement.
As a model system to study the luminescence properties of one-dimensional $c$-Si in the quantum-confinement regime we have chosen oxide-passivated layers. ${ }^{26}$ Thermal oxidation of silicon near $1000^{\circ} \mathrm{C}$ is known to produce a silicon surface having a low density of electronic defects and a surface oxide layer with band offsets in excess of $3 \mathrm{eV}$ for both conduction and valence electrons. A thermal oxide layer should therefore provide excellent passivation of the surface and confinement of charge carriers. Rapid thermal oxidation of anodically etched porous silicon to this temperature range produces material of slightly improved photoluminescence efficiency and superior photochemical stability compared to as-prepared porous silicon. ${ }^{27}$ Silicon nanocrystals passivated by a brief high-temperature oxidation achieve near unit radiative quantum efficiency at low temperature. ${ }^{2}$ Thus a highquality oxide passivation enables efficient photoluminescence in zero-dimensional silicon nanocrystals, and should facilitate an exploration of the luminescence properties of one-dimensional crystal silicon layers.

We have prepared thin Si layers surrounded by thermally grown $\mathrm{SiO}_{2}$ both from commercially prepared SIMOX (Ref. 28) silicon-on-insulator substrates and from crystallized $a$-Si:H layers deposited on both $a-\mathrm{SiO}_{2}$ and oxidized $c$-Si substrates. In all cases we observed red photoluminescence from $<2$-nm-thick silicon layers; the spectrum was a broad peak whose shape and peak wavelength were independent of the mean layer thickness.

\section{SAMPLE PREPARATION}

To produce a crystal silicon layer having a thickness of several nanometers or less, we oxidized silicon-on-insulator (SOI) substrates in flowing oxygen in a quartz tube furnace. SIMOX substrates were chosen over other SOI technologies because of the superior electrical properties of the upper silicon layer and the uniformity of the silicon layer thickness. SIMOX is prepared from a standard silicon substrate by a high-density, high-energy oxygen implant $\left(10^{-18} \mathrm{~cm}^{-2}\right.$, $190 \mathrm{keV}, T>600^{\circ} \mathrm{C}$ ) followed by a very high-temperature anneal $\left(1320^{\circ} \mathrm{C}, 6 \mathrm{~h}, \mathrm{~N}_{2}\right)$. This yields a slightly Si-rich $a-\mathrm{SiO}_{2}$ layer between the crystalline substrate and a $c-\mathrm{Si}$ upper layer of the same crystal orientation. ${ }^{29}$

The upper Si layer in the standard SIMOX wafers used in this study is $204.6 \mathrm{~nm}$ thick (3.0-nm peak-to-peak variation across a 4-in. diameter) and is separated from the $\mathrm{Si}(100)$ substrate by a $401.8-\mathrm{nm}$ buried oxide layer (4.6-nm peak-topeak variation). The upper silicon layer is essentially undoped, as the dopant atoms diffuse out of the layer during the long high-temperature anneal. Standard wafers are reported to have $<5 \times 10^{5} \mathrm{~cm}^{-2}$ threading dislocations in the upper Si layer and a pinhole density of $\sim 100 \mathrm{~cm}^{-2}$ in the oxide layer; a triple-implant wafer had a threading dislocation density $<1 \times 10^{4} \mathrm{~cm}^{-2}$. SOITEC SIMOX wafers have typical carrier lifetimes of a few tens of microseconds. "Low-dose" wafers were also used. They had a 180-nm Si layer over an 80-nm buried oxide layer. Oxidation at atmospheric pressure in a quartz tube furnace gradually converts the silicon layer into an upper oxide layer that is $\sim 2.25$ times as thick as the layer of silicon consumed. ${ }^{30}$ The sample is removed from the furnace to stop the oxidation just before the layer is entirely 
oxidized, leaving a thin $c$-Si layer surrounded by $\mathrm{SiO}_{2}$ on a Si substrate.

We found it useful to perform the 3-4 h of the oxidation in a spatial temperature gradient, producing a smooth variation in the thickness of the silicon layer. The taper impressed on the silicon layer thickness is somewhat reduced, but not eliminated, during subsequent oxidation under uniform temperature conditions. Because the electrical properties of the $\mathrm{Si} / \mathrm{SiO}_{2}$ interface are determined by the final oxidation temperature, this procedure ensured that the final $\mathrm{Si} / \mathrm{SiO}_{2}$ interface had uniform properties determined by the final temperature and atmosphere. Furthermore, recent work has shown that some oxygen diffuses through the silicon layer to the interface with the buried oxide, enough to grow a few nanometers of $\mathrm{SiO}_{2}$ at this interface. ${ }^{31}$ This ensures that both the upper and lower interfaces of the silicon layer have equivalent passivation. Typical Si layers were tapered $10 \mathrm{~nm}$ over $50 \mathrm{~mm}$.

\section{OPTICAL CHARACTERIZATION}

Reflection spectra were used to monitor the Si layer thickness. The interference of reflections from the four interfaces of high dielectric contrast present in these structures produces a spectrum that is highly sensitive to the thickness of the Si layer. Spectra were taken at an incidence angle of $\sim 9^{\circ}$ in a spectrophotometer over the wavelength range 205$800 \mathrm{~nm}(1.55-6.00 \mathrm{eV})$, and corrected by comparison to a scan of a thick $\mathrm{SiO}_{2}$ window, whose reflection spectrum was calculated from literature values of the refractive index. ${ }^{32}$ Spectra were typically made versus position along the sample with a beam width of $\sim 1.5 \mathrm{~mm}$, although for some a width of $\sim 1 \mathrm{~mm}$ was used. The spectra were fitted to a three-layer model ${ }^{33}$ that used bulk values for the dielectric functions of $c$-Si (Ref. 34) and $a-\mathrm{SiO}_{2}$ (Ref. 32) taken from the literature using standard least-squares techniques. ${ }^{35}$ This yielded the mean Si layer thickness versus position across a tapered sample.

Figure 1 shows such a sequence of spectra across a tapered SIMOX sample. The quality of the fits is quite good, especially when the Si layer thickness is not too small. Deviations at deep reflection minima arise primarily from thickness variations over the $\sim 1.5-\mathrm{mm}$ beam width. The right inset shows the thickness profile of the Si layer obtained from the fits, which agrees reasonably well with that calculated from the temperature profile of the furnace during sample oxidation. Since the beam samples macroscopic area of the sample, the thickness profile summarized in the inset represents the average Si layer thickness $d$ as a function of position $x$ across the taper.

For thicknesses less than a few nanometers, use of the bulk Si dielectric function tends to overestimate the layer thickness. The dielectric function of ultrathin silicon layers has recently been studied with spectroscopic ellipsometry and found to depart increasingly from the bulk dielectric function for thicknesses below $\sim 2 \mathrm{~nm}^{36}$ The influence on inferred thickness can be relatively large. For example, a fit using bulk dielectric values on a particular sample produced an estimated $\mathrm{Si}$ layer thickness of $0.5 \mathrm{~nm}$. Using the dielectric function for a 0.6-nm-thick $c$-Si layer reported in Ref. 36 gave a thickness of $1.2 \mathrm{~nm}$ (more than double) for the same

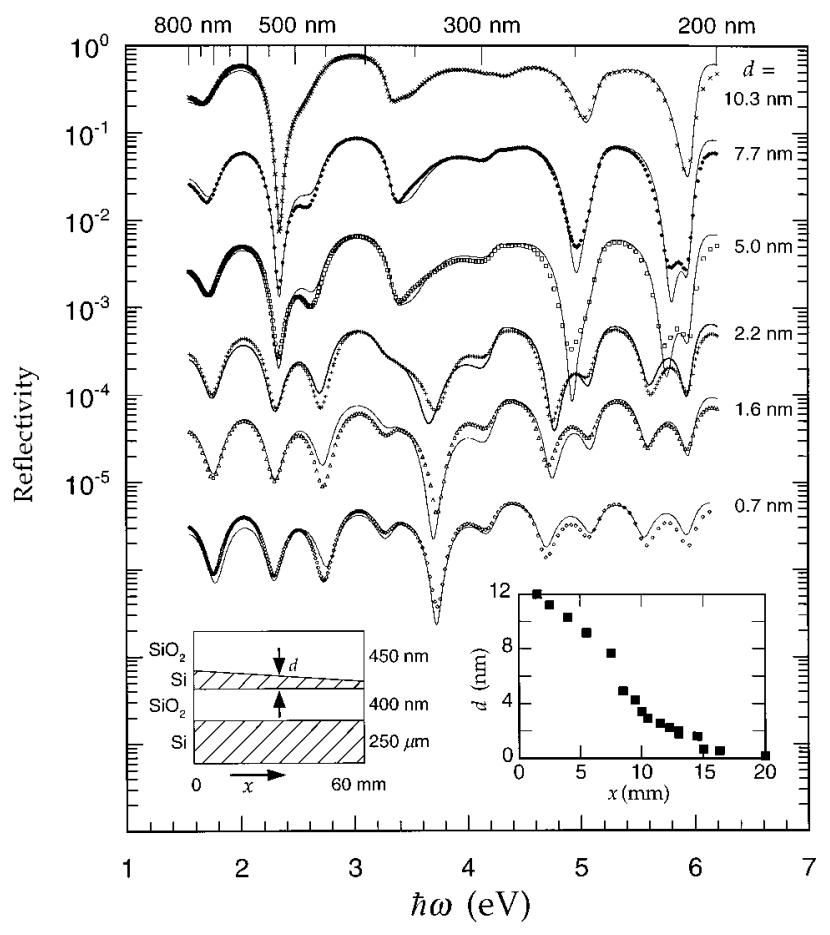

FIG. 1. Reflection spectra measured at several positions $x$ along the taper of a Si quantum-well layer made from a triple-implant SIMOX substrate, as illustrated in the left inset. Successive curves are offset by one order of magnitude for clarity. Symbols are data; smooth curves are fits to a three-layer model using bulk dielectric functions, yielding the layer thicknesses $d$ indicated at the right of each curve. The right inset shows the deduced thickness profile $d(x)$ across this sample.

sample. The latter value is undoubtedly more accurate, but is not self-consistent, as the deduced thickness $(1.2 \mathrm{~nm})$ is twice that for the dielectric function $(0.6 \mathrm{~nm})$. We have not yet implemented a self-consistent fitting algorithm to account for the thickness dependence of the dielectric function for ultrathin Si layers, and so we report here the values determined using bulk functions with the caveat that these values are underestimates for thicknesses less than $\sim 2 \mathrm{~nm}$.

Because of large thickness variations in the layers produced from SIMOX substrates, which will be discussed below, a means of making more uniform layers was pursued. Recent scanning tunnel microscope (STM) studies ${ }^{37}$ of thin $a$-Si:H films deposited on $c$-Si surfaces by PECVD of silane show that the rms roughness of the film grows quite slowly with film thickness and is small compared to that observed on SIMOX substrates. Therefore films much smoother than the SIMOX surface can be prepared by $a-\mathrm{Si}: \mathrm{H}$ deposition, provided that similar behavior is obtained for films deposited on $a-\mathrm{SiO}_{2}$ and that crystallization of the films does not cause significant roughening.

Hydrogenated amorphous silicon layers $\sim 20 \mathrm{~nm}$ thick were prepared by PECVD of pure silane in a radio-frequency discharge on fused silica and thermally oxidized $c-\mathrm{Si}$ substrates. ${ }^{38}$ The substrates were heated to $1000^{\circ} \mathrm{C}$ in flowing $\mathrm{O}_{2}$ in a quartz tube furnace for $\sim 5$ min immediately prior to insertion in the deposition chamber, to minimize surface contamination. Deposition was typically performed at $250{ }^{\circ} \mathrm{C}$; higher temperatures tended to produce films that 


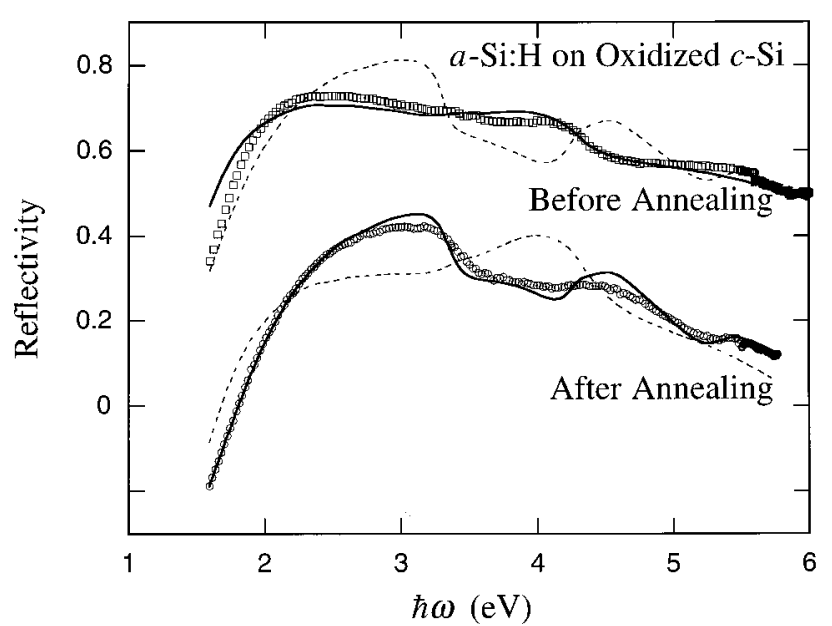

FIG. 2. Reflection spectra of crystallized $a-\mathrm{Si}: \mathrm{H}$ sample $D$ taken after annealing in Ar to $1000{ }^{\circ} \mathrm{C}$ and oxidation in 1 atm $\mathrm{O}_{2}$ at $1000{ }^{\circ} \mathrm{C}$ for $7 \mathrm{~min}$. Symbols are data; smooth curves are fits, which assume that the Si layer is fully crystalline.

flaked on annealing. The deposition rate was $0.1-0.2 \mathrm{~nm} / \mathrm{s}$ at a pressure of $65 \mathrm{~Pa}$. The films were crystallized in the quartz tube furnace by gradual heating from room temperature to $700-1000{ }^{\circ} \mathrm{C}$ in flowing Ar. Following this annealing treatment, the reflection spectrum of the film displayed a kink characterstic of $c$-Si at $\sim 3.4 \mathrm{eV}$, as shown in Fig. 2, demonstrating that the film had crystallized.

\section{PHOTOLUMINESCENCE MEASUREMENTS}

The $\mathrm{cw}$ and time-resolved photoluminescence properties of the Si layers were studied using either a $\mathrm{HeCd}$ laser operating at $325 \mathrm{~nm}(3.81 \mathrm{eV})$ or $442 \mathrm{~nm}(2.80 \mathrm{eV})$, or an air-cooled $\mathrm{Ar}^{+}$laser at $488 \mathrm{~nm}(2.54 \mathrm{eV})$, and a $\frac{1}{8}-\mathrm{m}$ double monochromator. Care was taken to reject background radiation from the laser plasma, to minimize photoinduced sample heating, and fluorescence saturation. The spectra were corrected for spectral sensitivity variations of the monochromator and photomultiplier.

All samples were observed to luminesce in the red and near infrared once the average thickness of the layer was small enough. An $\sim 0.003-\mathrm{cm}^{2}$ area of these samples was excited at $488 \mathrm{~nm}(2.54 \mathrm{eV})$ with a power of $2 \mathrm{~mW}$. The fluorescence intensity was proportional to excitation power under these conditions. Typical PL spectra of quantum-well layers made from SIMOX substrates and from crystallized $a$-Si:H layers on oxidized $c-\mathrm{Si}$ are shown in Fig. 3 , where they are compared to the spectrum of a stain-etched porous silicon sample. ${ }^{39}$ The spectra of the thin Si layers are similar, with peak energies of $1.62 \mathrm{eV}$ (SIMOX) and $1.58 \mathrm{eV}$ (crystallized $a-\mathrm{Si}: \mathrm{H})$ and full widths at half maximum (FWHM) of $0.23 \mathrm{eV}$. The porous silicon spectrum is a Gaussian centered at $1.74 \mathrm{eV}$ with FWHM equal to $0.32 \mathrm{eV}$, consistent with many published in the recent literature. It is also more than 100 times as intense as the fluorescence obtained here from the ultrathin Si layers.

Similar spectra and intensities were observed from samples made from standard single-implant SIMOX wafers (having $\mathrm{Si}$ islands in the buried oxide near the substrate), low-dose SIMOX (80-nm buried oxide and no buried is-

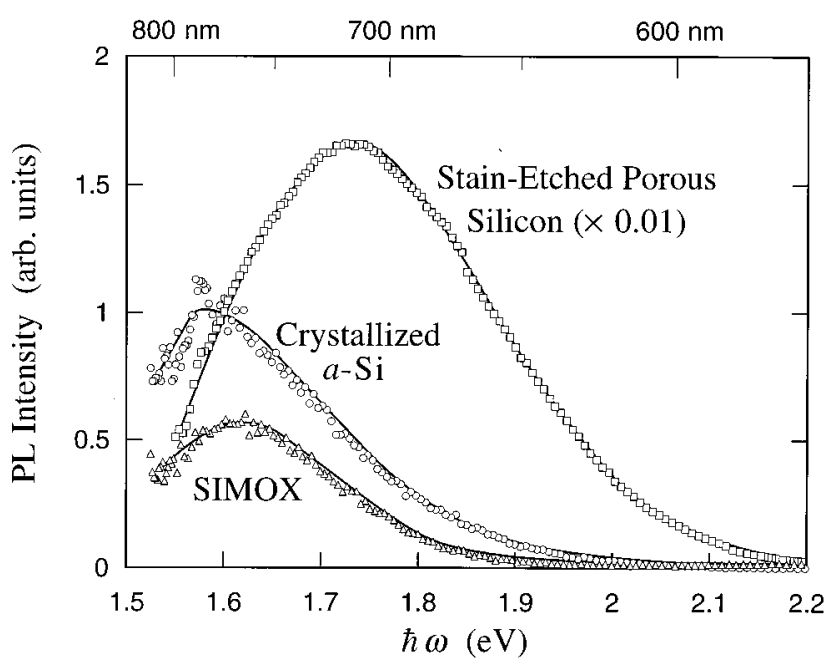

FIG. 3. Photoluminescence (PL) spectra of thin Si layers made from a SIMOX substrate $(\boldsymbol{\Delta})$, crystallized $a-\mathrm{Si}: \mathrm{H}$ deposited on an oxidized $c$-Si substrate $(\bigcirc)$, and of a stain-etched porous Si layer (ם). The excitation source was an $\mathrm{Ar}^{+}$laser $(488 \mathrm{~nm}, 2.54 \mathrm{eV})$ and the spectra were corrected for instrumental response. Smooth curves are guides to the eye.

lands), triple-implant SIMOX (400-nm buried oxide, no islands), and crystallized $a-\mathrm{Si}: \mathrm{H}$ layers grown on fused silica and on oxidized $c$-Si substrates. Furthermore, the spectral shape was independent of the average Si layer thickness on each sample, although its intensity varied smoothly with average thickness. This is illustrated in Fig. 4 which shows PL spectra of a Si layer prepared from standard SIMOX. This sample was prepared without a thickness taper; between successive spectra the sample was returned to the tube furnace for further oxidation at $1100{ }^{\circ} \mathrm{C}$, and then rapidly withdrawn. As the layer oxidized, the PL intensity was observed to rise more than two orders of magnitude without shifting appreciably. This appears in conflict with a simple quantumconfinement model, for which the confinement energy shift should increase with decreasing layer thickness. Subsequent spectra taken after further oxidation showed decreasing intensity, but no noticeable wavelength shift, and have been omitted from the figure for clarity.

Figure 5 shows the mean layer thickness $d$ and the observed $(750 \mathrm{~nm})$ PL intensity of the SIMOX well of Fig. 1. The peak intensity is observed for $d \approx 2 \mathrm{~nm}$, and the PL signal does not vanish for $d \approx 0$. Since absorption in the layer depends linearly on the layer thickness for very thin layers, the data in Fig. 5 imply that the PL efficiency increases monotonically with decreasing layer thickness.

The crystallized $a$-Si:H films showed even more remarkable PL dependence on layer thickness, a fact which we attribute to its smaller thickness variations. Figure 6 shows the mean layer thickness and the PL intensity of $a$-Si:H sample $D$ vs position $x$, analogous to Fig. 5 for the SIMOX layer. A representative reflection spectrum and fits are shown in Fig. 7 , taken at $x=7.5 \mathrm{~mm}$ and yielding a Si layer thickness of $0.5 \mathrm{~nm}$, assuming bulk values (solid curve), and $1.2 \mathrm{~nm}$, using the data for 0.6-nm $c$-Si from Ref. 36 (dashed curve). An intermediate value of $\sim 1 \mathrm{~nm}$ is probably reasonable. Regardless of the precise value of layer thickness at this position, the monotonic taper is preserved in the sample and the 


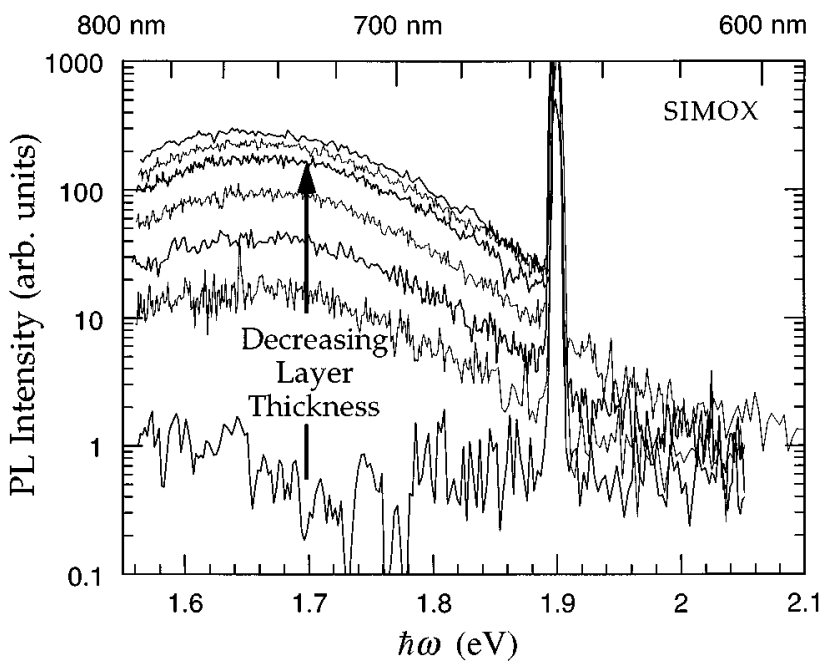

FIG. 4. PL spectra of a SIMOX sample excited with 325-nm light from a cw HeCd laser. No taper was impressed on this sample. Between successive spectra, the sample was returned to the quartz tube furnace in $\mathrm{O}_{2}$ flow at $1100{ }^{\circ} \mathrm{C}$ for $\sim 5 \mathrm{~min}$, which was sufficient to oxidize $1 \mathrm{~nm}$ of $\mathrm{Si}$, assuming standard oxidation kinetics. The upper curve shows the maximum light obtained from this sample. Additional spectra taken after further oxidation had the same shape but decreasing intensity. (The excitation beam was TE polarized and incident at $40^{\circ}$; the narrow peak at $1.9 \mathrm{eV}$ is the second-order diffraction peak of the scattered 3.8-eV pump light.)

reflection-determined layer thickness continues to vary linearly with position, as shown in open circles in Fig. 8, albeit at reduced slope. It extrapolates to 0 at $x \geqslant 9 \mathrm{~mm}$. However, paradoxically, this is precisely where strong luminescence is observed. This suggests that the luminescence arises from small Si crystallites which form as the layer breaks apart. Possibly, these crystallites cover too small a fraction of the total area to affect the reflection spectrum. Furthermore, once the film has broken into isolated regions it may oxidize more slowly due to the stress dependence ${ }^{40}$ of the oxidation rate. ${ }^{41}$

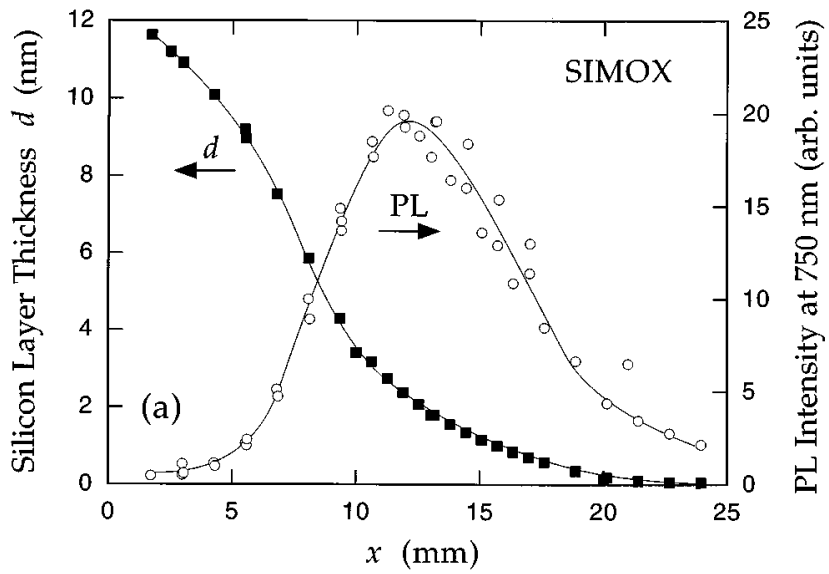

FIG. 5. Plot of the mean layer thickness $d$ and the peak PL intensity at $\lambda=750 \mathrm{~nm}$ as functions of position $x$ on the tripleimplant SIMOX sample of Fig. 2, showing a peak luminescence intensity for a thickness of $\sim 2 \mathrm{~nm}$. (b) Crystallized $a-\mathrm{Si}: \mathrm{H}$ layer on oxidized $c$-Si. Virtually no PL is observed in the region of nonnegligible thickness as seen in reflection spectra.

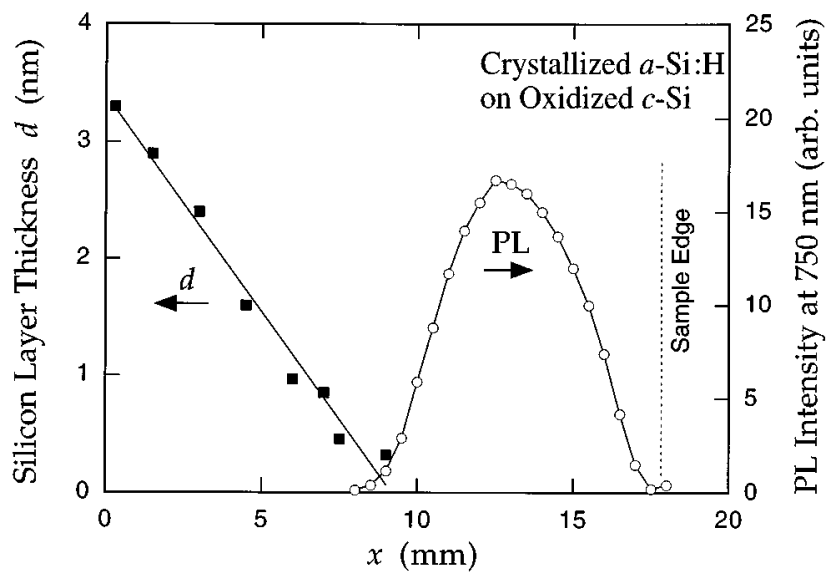

FIG. 6. Plot of the mean layer thickness $d$ and the peak PL intensity at $\lambda=750 \mathrm{~nm}$ as functions of position $x$ of $a$-Si:H sample $D$. Virtually no PL is observed in the region of non-negligible thickness, as determined from the reflection spectra.

\section{MORPHOLOGY OF THIN SILICON LAYERS}

To investigate the thickness variations of the Si layer, two forms of microscopy were used. Thickness variations on the scale of several micrometers were studied by scanning a tightly focused HeNe laser spot over the sample and recording variations in the reflected intensity. Assuming the variation in reflectivity arises from thickness variations in the silicon layer (as opposed to scattering), the change in reflected intensity is proportional to the change in the silicon layer thickness. A $100 \times$ microscope objective was used (spot size $\approx 0.9 \mu \mathrm{m}$ ) to focus the HeNe beam, which was scanned over a $\sim 25-\mu \mathrm{m}$ range on the sample surface. From the reflectivity variations an $\sim 1.5$-nm peak-to-peak variation in SIMOX layer thickness, typically with a period of $2-3 \mu \mathrm{m}$, was inferred in regions where $d \gtrsim 3 \mathrm{~nm}$.

Optical methods have the advantage that they are nonde-

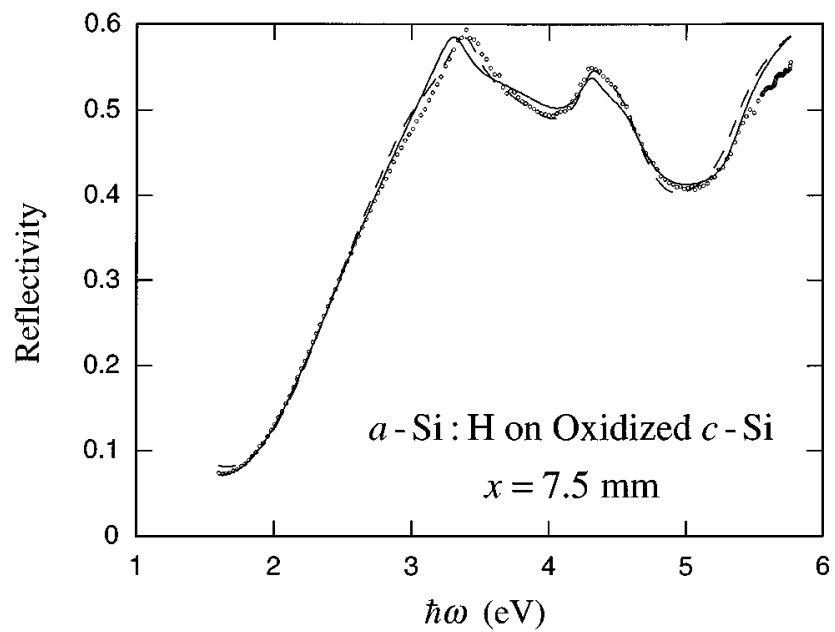

FIG. 7. Reflection spectrum at $x=7.5 \mathrm{~mm}$ on $a-\mathrm{Si}: \mathrm{H}$ sample $D$. The solid curve is a fit using the index of refraction of bulk $c$-Si, giving an estimated thickness of $0.5 \mathrm{~nm}$. The dashed curve fit is a fit using the dielectric function of 0.6-nm-thick $c$-Si taken from Ref. 36, which gives $1.2 \mathrm{~nm}$ for the Si layer thickness. 


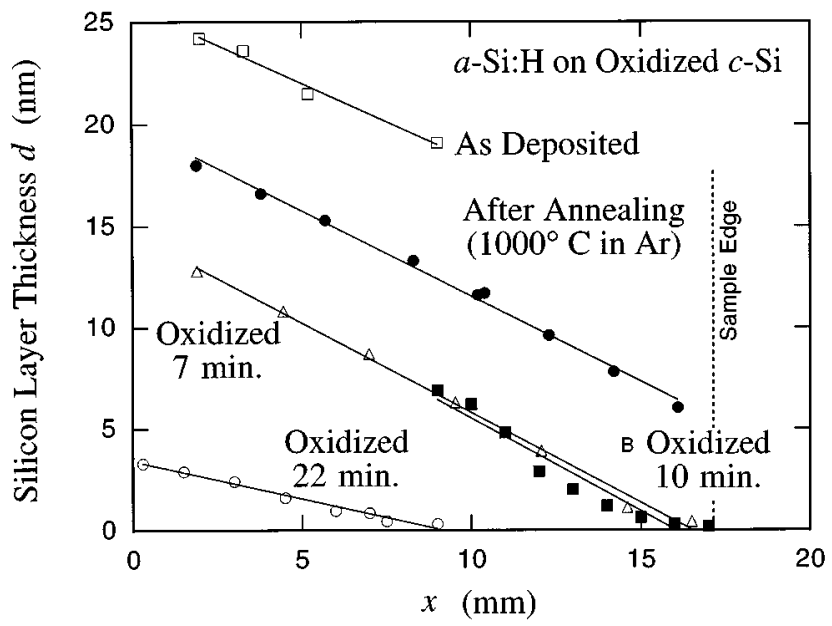

FIG. 8. Silicon layer thickness profiles of $a$-Si:H sample $D$ taken after $a$-Si:H deposition ( $\square$ ), after annealing/crystallization (○), after $7 \mathrm{~min}$ in $\mathrm{O}_{2}$ at $1000^{\circ} \mathrm{C}(\triangle)$, after a total of $10 \mathrm{~min}$ of oxidation ( $\boldsymbol{\square})$, and after a total of $22 \mathrm{~min}$ of oxidation $(\bigcirc)$.

structive and sensitive to subnanometer thickness variations, but the disadvantage of limited spatial resolution. Higher resolution is required for these very thin layers. The electrical isolation provided by the buried oxide causes charging problems in electron microscopy; scanning electron microscope images had poorer contrast and resolution than did optical micrographs. The charging problem also prevents using scanning tunneling microscopy. Cross-sectional transmission electron microscopy (TEM) can provide valuable information on the thickness and crystallinity of the Si layer, but was not readily available. One sample was investigated by TEM, which confirmed the presence of a thin crystalline layer approximately $2 \mathrm{~nm}$ thick, in agreement with reflectivity data.

Atomic force microscopy (AFM) images of several samples were made after removal of the upper oxide layer with buffered HF. HF etches Si extremely slowly, ${ }^{42}$ so that after a brief HF etch, the upper oxide is removed but the $\mathrm{Si}$ layer remains unmodified. Both $\mathrm{Si}$ and silicon nitride tips were tried; the best results were obtained with silicon nitride tips in contact mode. These tips have a typical radius of 40 $\mathrm{nm}$. We assume that deviations from flatness of the upper surface correlate with variations in the layer thickness.

The images were analyzed by removing the plane, then calculating the root-mean-square surface height variation (rms roughness). In addition, the height correlation function $g_{x}(d)$ was calculated along the scan direction $x$ for representative areas of the image from the expression

$$
g_{x}(k s)=\frac{\sum_{i=1}^{n} \sum_{j=1}^{m-k}\left(h_{i j}-\bar{h}\right)\left(h_{i, j+k}-\bar{h}\right)}{n(m-k)},
$$

where $h_{i j}$ is the height of the surface at $(i, j), s$ is the distance between measurements, and $\bar{h}$ is the mean height. The value of $k s \equiv d_{1 / 2}$ at which $g_{x}$ is reduced to half its peak value was used as a measure of the lateral extent of surface features.

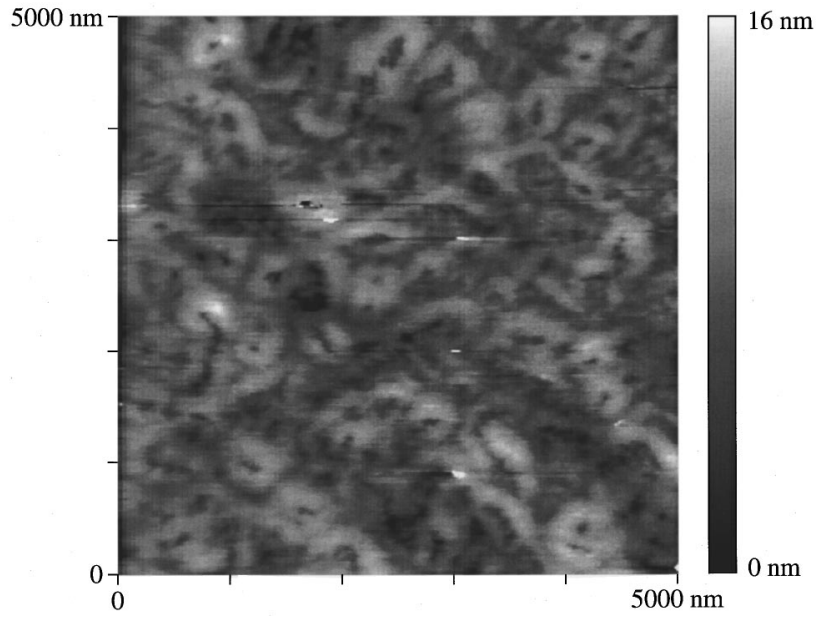

FIG. 9. AFM image of a $5 \mu \mathrm{m} \times 5 \mu \mathrm{m}$ region of a SIMOX sample made when the average Si layer thickness measured by reflectivity was $\sim 10 \mathrm{~nm}$. The rms roughness of this image is $\sigma=2.25 \mathrm{~nm}$.

\section{SIMOX layers}

The surface of a 10-nm-thick portion of a Si layer made from standard SIMOX is shown in Fig. 9. It has a root-meansquare roughness of $2.3 \mathrm{~nm}$, exceeding by an order of magnitude the roughness of a commercial $\mathrm{Si}(100)$ wafer and the value reported for thermally oxidized $\mathrm{Si} / \mathrm{SiO}_{2}$ interfaces (see Table I) ${ }^{43,44}$ Furthermore, the correlation length of the surface in the scan direction is $d_{1 / 2} \approx 130 \mathrm{~nm}$. This is much larger than the correlation length of a $c$-Si wafer surface measured with the AFM $(25 \mathrm{~nm})$, a value that is inflated by the low resolution of the image. TEM images of the $\mathrm{Si} / \mathrm{SiO}_{2}$ interface give correlation lengths of $\sim 4 \mathrm{~nm}$ (Ref. 44) and a STM image of a $c$-Si surface in UHV, taken in our laboratory, gave $d_{1 / 2}=2.2 \mathrm{~nm}$. This large "corrugation" of the Si surface in SIMOX has been described by Sato and Yonehara, ${ }^{45}$ and attributed to the lengthy high-temperature anneal to which the wafer is subjected after implantation, which forms a (near) stoichiometric $\mathrm{SiO}_{2}$ layer. At $1320^{\circ} \mathrm{C}$, the oxide layer is quite plastic and fluctuations in the thickness of the oxide and of the upper Si layer become frozen in upon cooling.

As oxidation continues the surface begins to break apart when the thinnest regions are entirely consumed. This can be seen quite clearly in Fig. 10, which shows the surface of the

TABLE I. Root-mean-square roughness $\sigma$ and correlation length $d_{1 / 2}$ of various surfaces as determined from AFM measurements. On the two asterisked surfaces the correlation length was overestimated due to insufficient image resolution. The low resolution did not affect the roughness.

\begin{tabular}{lcc}
\hline \hline \multicolumn{1}{c}{ Surface } & $\sigma(\mathrm{nm})$ & $d_{1 / 2}(\mathrm{~nm})$ \\
\hline Crystal silicon & $0.12^{*}$ & 25 \\
SIMOX well, $10 \mathrm{~nm}$ thick & 2.25 & 130 \\
$a-\mathrm{Si}: \mathrm{H}$ on oxidized $c-\mathrm{Si}$ & 0.43 & 4 \\
Crystallized $a$-Si:H on oxidized $c-\mathrm{Si}$ & $0.4^{*}$ & 13 \\
2-nm crystallized $a$-Si:H on oxidized $c-\mathrm{Si}$ & 0.53 & 8 \\
1-nm crystallized $a$-Si:H on oxidized $c-\mathrm{Si}$ & 0.65 & 5.7 \\
\hline \hline
\end{tabular}




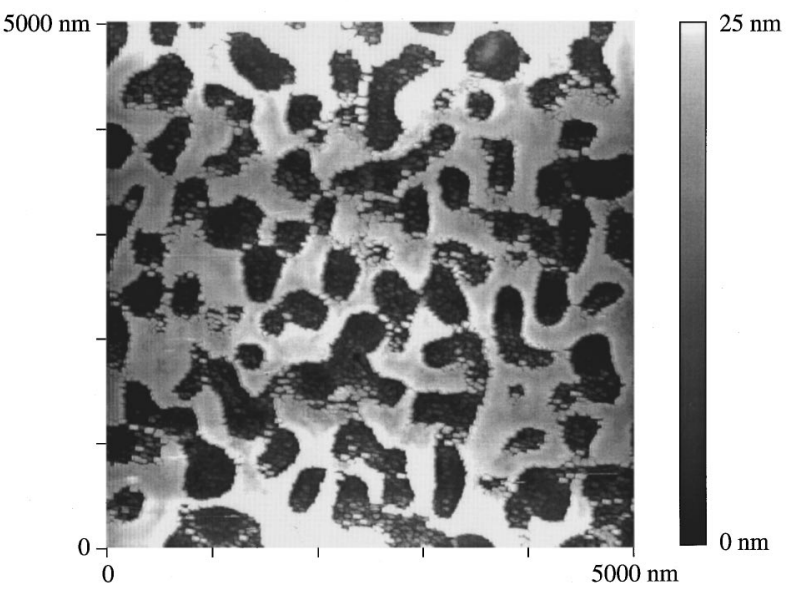

FIG. 10. AFM image of a $5 \mu \mathrm{m} \times 5 \mu \mathrm{m}$ portion of the luminescing region of a SIMOX sample following removal of the upper oxide with buffered oxide etch. The large pits in the image arise from regions in which the entire thickness of the Si layer had been oxidized, allowing the etchant to attack the buried oxide.

same sample in a region that luminesced $(d \approx 1-2 \mathrm{~nm})$. This "surface" consists of the Si layer perforated with deep holes $\sim 300 \mathrm{~nm}$ in diameter. Where the Si layer has been entirely oxidized, the HF used to remove the upper oxide layer etches deep pits in the exposed buried oxide. Archipelagos of smaller $\mathrm{Si}$ islands appear to decorate the perimeter of these holes. Their size is roughly $30-100 \mathrm{~nm}$, although the resolution of the AFM image, particularly near large vertical steps, does not permit an accurate determination of their size and density. It is possible that these small isolated regions produce the photoluminescence and that the rest of the structure is essentially dark. However, the large thickness variations in SIMOX layers make it difficult to correlate photoluminescence properties and size, which prompted us to investigate crystallized $a-\mathrm{Si}: \mathrm{H}$ films.

\section{Crystallized $a$-Si:H films}

AFM images of crystallized $a$-Si:H films were made at each step of the growth process to clarify the structure of the thin films. Typical oxide layers grown on $c$-Si substrates at $1000{ }^{\circ} \mathrm{C}$ in dry $\mathrm{O}_{2}$ were $100 \mathrm{~nm}$ thick. Measurements of the oxide surface were unreliable, perhaps due to charging effects in the (thick) oxide, and gave unrealistically high roughness $(\sigma \approx 1 \mathrm{~nm})$. Measurements on $a$-Si: $\mathrm{H}$ films on thick oxide did not suffer these problems and yielded $\sigma=0.43 \mathrm{~nm}$ and $d_{1 / 2}=4 \mathrm{~nm}$. These values are in good agreement with STM measurements of $a-\mathrm{Si}: \mathrm{H}$ film surfaces, grown on $c$-Si substrates with the same deposition system in our laboratory. For this same 20-nm film thickness, the STM data yielded $\sigma \approx 0.46 \mathrm{~nm}$ and $d_{1 / 2} \approx 3.5 \mathrm{~nm}$. The agreement with the AFM results is remarkable, especially considering the larger (nominal 40-nm) AFM tip radius, and it indicates an excellent resolution for the AFM. Furthermore, the annealing and crystallization caused no significant change in the roughness of the $\mathrm{Si}$ films; $\sigma$ remained $0.4 \mathrm{~nm}$.

After oxidation to produce a tapered layer, the upper oxide was removed with HF and the H-terminated layer surface was imaged with the AFM. Where the (reflectivity-

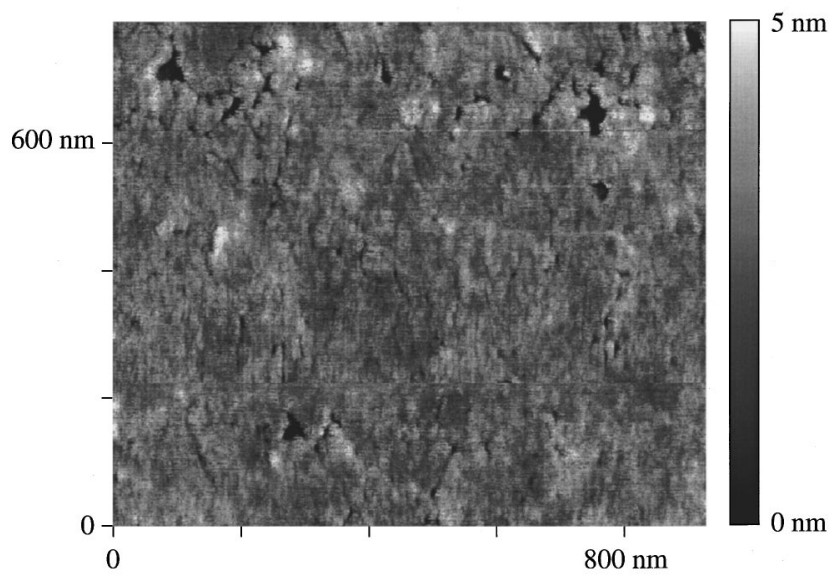

FIG. 11. AFM image of a $1 \mu \mathrm{m} \times 1 \mu \mathrm{m}$ portion crystallized $a$-Si:H layer that was thinned by oxidation at $1000{ }^{\circ} \mathrm{C}$. The mean layer thickness determined from reflection spectra was $\sim 1 \mathrm{~nm}$ in the imaged region.

determined) mean layer thickness was $2 \mathrm{~nm}$, the film appeared smooth and continuous, with $\sigma=0.53 \mathrm{~nm}$ and $d_{1 / 2}=8 \mathrm{~nm}$. This roughness is only slightly greater than before oxidation, but the correlation length is roughly double that of the unoxidized film. No luminescence was observed from this region of the film.

Figure 11 shows the surface of the $\mathrm{Si}$ layer where the mean thickness (assuming bulk $c$-Si) was only $1 \mathrm{~nm}$, suggesting an actual thickness between 1 and $2 \mathrm{~nm}$. Several small dark pits with a characteristic size of $\sim 10 \mathrm{~nm}$ are clearly visible and correspond to holes in the layer. Their density was estimated to be $\sim 100 / \mu \mathrm{m}^{2}$. Several larger pits, up to $\sim 50 \mathrm{~nm}$ in size, are also evident in the $1-\mu \mathrm{m}^{2}$ image. At this mean thickness, the layer is still continuous, although it shows signs of breaking apart. Roughness has increased to $0.65 \mathrm{~nm}$ in regions without large pits; therefore this represents an upper bound on the roughness, which has increased appreciably compared to the thicker film, but remains four times smaller than that of the SIMOX film. Despite the small average thickness and thickness variation, this film did not luminesce. Nonetheless, estimates of the quantumconfinement energy shift made by scaling results using the linear combination of atomic orbitals technique for nanowires ${ }^{46}$ suggest that the direct band gap of a 1-nm-thick layer should be $\sim 2 \mathrm{eV}$ and hence visible to our apparatus. An AFM image of the luminescing region of the film is shown in Fig. 12; it appears to be divided into isolated, roughly round regions $\sim 50-100 \mathrm{~nm}$ in size. The rms roughness of this surface is $2.6 \mathrm{~nm}$, which likely reflects the higher density of pits in the substrate oxide. Reflectivity measurements of the sample in this region, prior to removal of the upper oxide, gave a negligible thickness for the Si layer, suggesting a strong variation of the dielectric properties of ultrathin Si layers compared to bulk properties and that just enough Si remains in the layer to arrest etching.

\section{DISCUSSION}

We have observed similar photoluminescence behavior from SIMOX and crystallized $a$-Si:H layers passivated on both sides by high-temperature oxide. The main features of 
Luminescent Region of Crystallized $a$-Si:H Layer

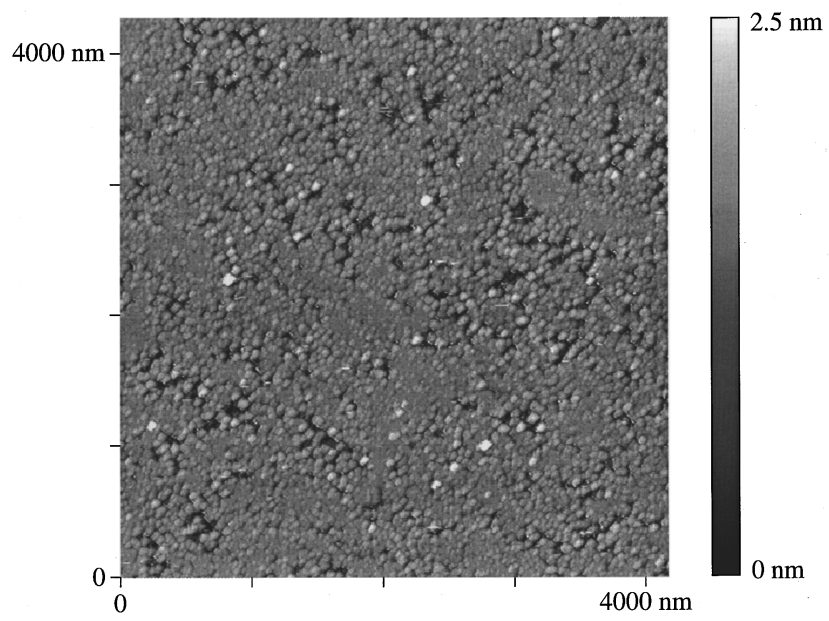

FIG. 12. AFM image of a $5 \mu \mathrm{m} \times 5 \mu \mathrm{m}$ area in the luminescent region of the sample shown in the previous figure.

this luminescence are as follows. SIMOX layers with average thickness greater than $10 \mathrm{~nm}$ and crystallized $a-\mathrm{Si}: \mathrm{H}$ layers with average thickness greater than $1 \mathrm{~nm}$ emit no detectable light, but as they are oxidized further they begin to luminesce in a broad peak in the near-infrared and red portion of the spectrum. The peak is centered near $1.65 \mathrm{eV}$, which is at least $0.1 \mathrm{eV}$ lower than typical porous silicon spectra. As a luminescing sample is further oxidized, the PL intensity first increases then decreases and vanishes, yet the peak wavelength and width remain essentially constant. Rapid withdrawal of a sample from flowing $\mathrm{O}_{2}$ at $1100{ }^{\circ} \mathrm{C}$ produces the greatest PL efficiency; slow withdrawal, annealing in an inert gas, and oxidation at lower temperature all reduce the PL efficiency.

We consider first in what respect this system differs from previously investigated luminescing silicon systems, and then turn to possible explanations of these observations and mechanisms for the luminescence. Prior to oxidation, the SIMOX layer is certainly crystalline; oxidation, even at high temperature, does not render crystal silicon amorphous. Furthermore, a 2-nm-thick layer appeared crystalline in TEM. We conclude, therefore, that the SIMOX Si quantum layers are composed of crystal silicon. A similar conclusion for the crystallized $a-\mathrm{Si}: \mathrm{H}$ films is supported by the reflectivity change observed following annealing and by the similarity of PL behavior between the SIMOX wells and the crystallized $a$-Si:H wells.

Even upon breakup of the layers, the lateral dimensions of most structures appear much larger than the thickness, suggesting that confinement is predominantly one dimensional, although it is perhaps possible that the luminescent fraction is confined in all three dimensions. These layers are distinct from Si-rich oxide layers in which amorphous silicon precipitates were observed to electroluminesce. ${ }^{9}$ They are also different from $a$-Si:H films containing $\mathrm{Si}$ nanocrystals and from oxidized Si nanocrystals due to the predominantly onedimensional nature of the carrier confinement. Crystallized amorphous multilayers ${ }^{5,6,8}$ consist of roughly spherical crystallites-whose diameter is determined by the layer thickness-surrounded by amorphous material. As such, they are similar in geometry to Si nanocrystals, although the details of the surface may be entirely different. The layers described here, particularly those made by crystallizing $a-\mathrm{Si}: \mathrm{H}$, are quite flat and two dimensional, as can be seen by comparing the lateral correlation length to the rms roughness.

The AFM results show that the thickness variations of the $\mathrm{Si}$ layer produced by oxidizing crystallized $a-\mathrm{Si}: \mathrm{H}$ on thermally grown $\mathrm{SiO}_{2}$ are much smaller than those of the layers produced from SIMOX substrates, and that in the luminescing region the layer appears segmented into isolated regions approximately $50-100 \mu \mathrm{m}$ in diameter. With increasing oxidation both SIMOX and crystallized a-Si:H layers have virtually identical PL spectra, and produce similar levels of luminescence, although they differ in the mean thickness-as measured by reflectivity - that produces the peak signal.

The temporal behavior of SIMOX Si layers was investigated on the microsecond time scale by chopping the 488-nm excitation beam with an acousto-optic modulator. At room temperature, the initial PL decay time was $\sim 35 \mu \mathrm{s}$, although the decay was nonexponential. Nonexponential decay of the PL signal from porous silicon and Si nanocrystallites has been widely reported. ${ }^{2,3,47}$ Surprisingly, the PL decay rate was observed to be independent of wavelength within the broad PL band. This is in contrast to the spectral diffusion observed in porous silicon, ${ }^{3,48,49}$ in which the blue side of the peak decays more rapidly than the red.

The cw photoluminescence results are similar to the slow red PL band in porous silicon and, together with the observed $\sim 35-\mu$ s PL decay time, indicate that the emitted red/ near-infrared (IR) light comes from thermalized carriers excited in the Si layer. An estimate of the PL efficiency of the SIMOX layer was made assuming a uniform layer of thickness equal to the value determined from fits to the reflection spectrum. The model used the bulk $c$-Si index of refraction and absorption coefficient for the silicon layer, and the calculation of the absorbed laser fluence took account of reflections in the multilayer structure. The estimate of $<10^{-4}$ at room temperature is low compared to $1-10 \%$ reported for some porous silicon ${ }^{50}$ and oxidized Si nanocrystals. ${ }^{13}$ However, it appears very likely that the large thickness variations of the SIMOX well, reflected in the rough upper surface of the $\mathrm{Si}$ layer, make large regions of the layer too thick to luminesce, and that the observed light is generated by a very small fraction of the layer. The fact that the crystallized $a$-Si:H layer produced roughly the same intensity of photoluminescence, yet had negligible thickness, shows that the efficiency of a layer can be much higher, perhaps approaching that of porous silicon. The low signal level in our case results from the very small volume of luminescent material. $^{51}$

The luminescence behavior of the layers appears inconsistent with a strict one-dimensional quantum-confinement model, since the spectrum of both SIMOX and crystallized $a$-Si:H wells was independent of mean layer thickness. Similar behavior has been observed in mesoporous silicon as it is oxidized. $^{52}$

The PL intensity, however, does depend on thickness. When the layer is continuous and thin (see Fig. 11), no PL is observed. When the layer has oxidized further, it appears as 
a fairly regular array of 50-100-nm "pancakes" (Fig. 12). This size is two to four times the initial $a$-Si:H layer thickness and may represent the size of microcrystals formed during annealing. Rapid thermal annealing of $a-\mathrm{Si}: \mathrm{H}$ films on oxide substrates has been observed to produce high-quality $c$-Si films whose mean crystallite size exceeds the layer thickness. ${ }^{53}$ It is reasonable to expect that grain boundaries will oxidize more rapidly than the crystallites themselves; because they contain a high density of defective bonds and fast recombination centers, until they do oxidize, they must effectively suppress luminescence. Efficient luminescence may only result when small enough regions of the film are effectively isolated. Assuming that the mean density of midgap states at the $\mathrm{Si} / \mathrm{SiO}_{2}$ interface is $10^{14} \mathrm{~m}^{-2}$, typical of high-quality thermal oxide, the probability that a $50-\mathrm{nm}$ diam $\mathrm{Si}$ pancake has no midgap states is $60 \%$. Thus if they have radiative centers a great many of them should luminesce.

A further test of the quantum-confinement hypothesis is possible by comparing the luminescence spectrum of layers with different crystallographic orientation. Because the conduction-band minimum is near the $\mathrm{Si} X$ point, and because the $X$ valleys are highly anisotropic, with transverse mass $m_{t}=0.19 m_{0}$ and longitudinal mass $m_{l}=0.98 m_{0}$, the confinement energy shift of the conduction band depends on the orientation of the layer with respect to the crystallographic axes. For (001) SIMOX, the effective mass along the confinement direction for the (100) and (010) valleys is $m_{t}$, leading to a large shift. However, the (001) valleys have mass $m_{l} \gg m_{t}$ and therefore shift much less. Since carriers scatter among the conduction valleys by emitting momentum-conserving phonons on a picosecond time scale, the effective band-gap increase is governed by the lowestlying valleys. In the effective mass approximation it is proportional to $m^{-1}$, where $m$ is the largest mass. For a layer oriented along (110), the effective masses are

$$
\frac{1}{m}=\left\{\begin{array}{l}
\frac{1}{2}\left(\frac{1}{m_{t}}+\frac{1}{m_{l}}\right) \approx \frac{1}{2 m_{t}}, \quad(100) \text { and (010) valleys } \\
\frac{1}{m_{t}}, \quad(001) \text { valleys }
\end{array}\right.
$$

while for a (111) oriented layer, all valleys have the same mass,

$$
\frac{1}{m}=\frac{1}{6}\left(\frac{2}{m_{t}}+\frac{1}{m_{l}}\right) \approx \frac{1}{3 m_{t}} .
$$

Hence the confinement energy shifts for the different layers should be in the proportion $1: 1.72: 2.58$ for the orientations (100) : (111) : (110).

SIMOX substrates with a $\sim 200$-nm Si layer over a $\sim 400$-nm buried oxide were prepared from $\mathrm{Si}(110)$ and $\mathrm{Si}(111)$ wafers by SOITEC Inc. and oxidized in the usual way to yield a tapered Si layer. Figure 13 shows the PL spectra from these samples compared to that from the standard (100) SIMOX. The (110) and (111) wafers had nearly identical spectra that were slightly redshifted compared to (100) SIMOX. This is opposite to the trend expected from quantum confinement.
The $\mathrm{Si} / \mathrm{SiO}_{2}$ interface with the lowest defect density is obtained with $\mathrm{Si}(100) .{ }^{54}$ Interfaces oriented along (110) and (111) have greater densities of states throughout the band gap, and near the band edges in particular. If we extrapolate the rising density of states near the band edges to higher energy-where they are normally obscured by the bulk bands but are exposed by quantum confinement in nanoscale silicon-we infer a high density of localized states that is presumed responsible for the efficient PL of porous silicon in the surface-state model. In a surface-state model in which charges are rapidly trapped in localized states and migrate over only limited distances before recombining, the greater density of near-band-edge states on (110) and (111) surfaces could allow trapped charges to sample a greater number of localized states and settle to lower energy on average before recombining, thereby producing a redshifted spectrum. ${ }^{55}$

Additional evidence for the surface-state mechanism comes from the PL behavior of the samples with the upper oxide removed. Removing the upper oxide layer by immersing the sample in buffered HF caused a pronounced shift of the luminescence spectrum to higher energy for samples made from both SIMOX and crystallized $a-\mathrm{Si}: \mathrm{H}$, as seen in Fig. 14. In both cases the blueshift was $\sim 300 \mathrm{meV}$ and the fluorescent intensity was essentially unchanged. Removing the oxide from thick layers that did not luminesce before its removal failed to produce luminescence.

Buffered HF produces hydrogen-terminated Si surfaces which are very effectively passivated. Freshly prepared porous silicon is hydrogen terminated and luminesces strongly, so it is not surprising that a $\mathrm{Si}$ layer with hydrogen passivation on one side luminesces. The roughly constant spectrally integrated PL intensity suggests that hydrogen termination does not produce a significant increase in nonradiative recombination centers, consistent with oxidation studies of porous silicon. ${ }^{27}$ The magnitude of the spectral shift, however, is difficult to explain in a quantum-confinement model. A simple envelope-function calculation of the confinement energy increase caused by replacing an oxide barrier with vacuum gives less than one-tenth the observed shift. More detailed calculations on dots give a weaker dependence on size ${ }^{56}$ suggesting that if anything, the envelope-function estimate constitutes an upper bound. If the HF etch selectively removed certain crystallites, one might observe a shift in the spectral peak. However, one would expect the smaller crystallites to be more readily removed and this would lead to a redshifted spectrum if the quantum-confinement hypothesis were correct. More importantly, Fig. 14 shows an increase by more than an order of magnitude in the PL for $\hbar \omega>1.85 \mathrm{eV}$, which is inconsistent with removal of some of the luminescent material.

The blueshift perhaps arises from relaxation of the surface $\mathrm{Si}$ layer on replacement of the $\mathrm{SiO}_{2}$ layer with $\mathrm{H}$ termination. Although the Si layer is not strained by cooling-because it is the same material as the (effectively infinitely thick) substrate-the interface between $\mathrm{Si}$ and $\mathrm{SiO}_{2}$ does have stresses associated with the different bond lengths and angles of $\mathrm{Si}$ atoms in the two materials, as well as their different coefficients of thermal expansion, which produces stress on cooling. Replacement of the oxide with hydrogen at room temperature could thus be expected to give rise to a different 


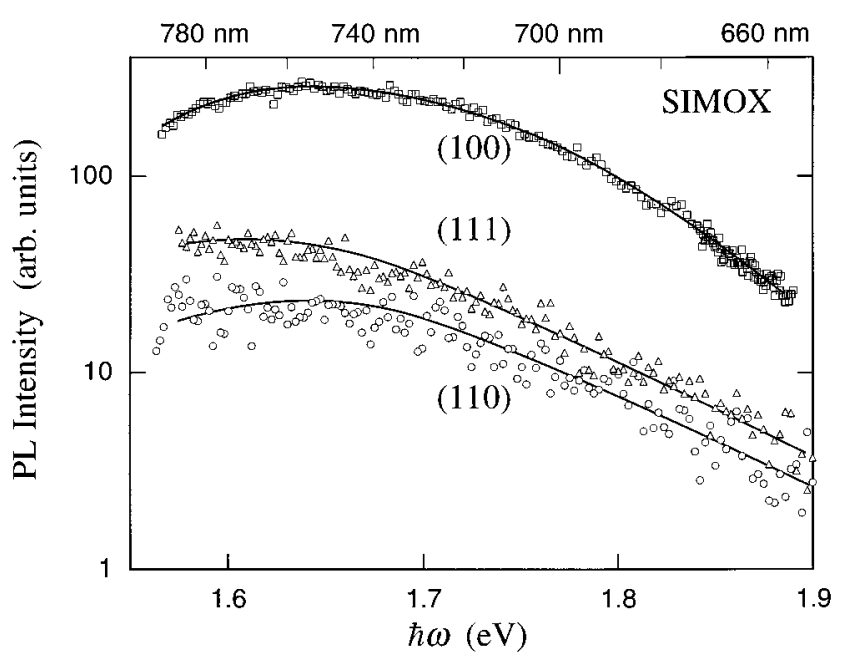

FIG. 13. PL spectra showing the influence of crystallographic orientation. Samples with tapered Si layers were prepared from (100), (110), and (111) SIMOX substrates with 200-nm initial Si layers over 400 -nm buried oxide layers. The (100) layer is blueshifted compared to the (110) and (111) layers, which is contrary to the shift expected for a quantum-confinement model.

distribution of states at the upper silicon surface and, assuming radiative recombination occurs through these states, a different PL spectrum.

\section{CONCLUSION}

We have prepared thin crystal silicon quantum-well layers by high-temperature dry oxidation of silicon on insulator substrates. The substrates were commercial SIMOX wafers and PECVD $a$-Si:H layers deposited on oxidized $c$-Si and fused silica substrates, which were crystallized in a quartz tube furnace. The crystallinity of the layers was confirmed by spectral reflectivity and TEM observation. Layers that were sufficiently thinned by oxidation emitted a broad red photoluminescence peak centered at $\sim 1.65 \mathrm{eV}$ that was independent of the mean layer thickness. The intensity of this luminescence decreased on annealing at high temperature in an inert gas and was restored, though not significantly increased, by annealing in hydrogen. The luminescence spectrum was largely unaffected by these treatments, but it blueshifted $\sim 0.3 \mathrm{eV}$ on removing the upper oxide with $\mathrm{HF}$. Under pulsed excitation, the photoluminescence decayed in a nonexponential manner with a first $e$-folding time of $\sim 35 \mu \mathrm{s}$ at room temperature, and slightly longer at $88 \mathrm{~K}$. The luminescence decay rate at a given temperature was independent of PL wavelength within the luminescence band. Layers with an average thickness of $\sim 1-1.5 \mathrm{~nm}$ and $\mathrm{rms}$ roughness of $0.4 \mathrm{~nm}$ did not luminesce. All properties appear inconsistent with a quantum-confinement model of nc-Si, in which electron-hole recombination is assumed to take place

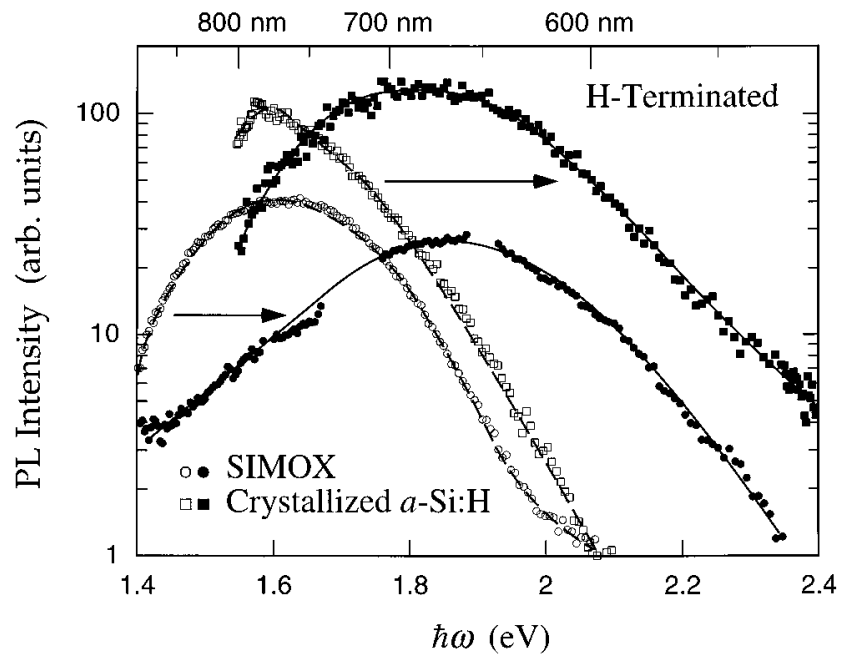

FIG. 14. PL spectra taken following the removal of the upper oxide layer with $p \mathrm{H}$-buffered $\mathrm{HF}$ (filled symbols) are shifted $\sim 0.3$ $\mathrm{eV}$ to higher energy compared to those taken with the oxide layer intact (open symbols). The same behavior was observed with SIMOX samples (squares) and crystallized $a-\mathrm{Si}: \mathrm{H}$ layers (circles).

in (fully) passivated Si structures, and they suggest the importance of radiative and nonradiative recombination at surface states. They do suggest, however, that quantum confinement is essential to produce luminescence because only layers thinner than several nanometers are found to luminesce.

The morphology of the layers was studied with an atomic force microscope, which revealed large-scale roughness in the SIMOX layers and significantly lower roughness on crystallized $a$-Si:H layers. The quantum efficiency of luminescence of rough layers $(\sigma \approx 2.3 \mathrm{~nm})$ was very low $\left(\sim 10^{-4}\right)$, but thin layers of lower roughness, when broken into regions of 50-100-nm diameter, luminesced with significantly higher efficiency. This size scale is compatible with a large fraction of regions having no midgap defects, which are likely responsible for nonradiative recombination. We conclude that room-temperature luminescence is possible in planar crystal silicon nanostructures, provided that nonradiative recombination at midgap states can be effectively suppressed.

\section{ACKNOWLEDGMENTS}

We are pleased to acknowledge the assistance of Jon Moore and Kenneth Douglas with the AFM images, Mike Estes, Garret Moddel, Jacques Pankove, and Edith Molenbroek for useful discussions, and Arnaldo Laracuente and David Tanenbaum for help with the amorphous silicon depositions. P.N.S. is grateful for support from the National Research Council.
*Present address: Physics Department, Harvey Mudd College, Claremont, CA 91711. Peter-Saeta@hmc.edu

${ }^{\dagger}$ Also at Quantum Physics Div., NIST, Boulder, CO 80309-0440.

${ }^{1}$ L. T. Canham, Appl. Phys. Lett. 57, 1046 (1990).

${ }^{2}$ K. A. Littau, P. J. Szajowski, A. J. Muller, A. R. Kortan, and L.
E. Brus, J. Phys. Chem. 97, 1224 (1993); L. Brus, ibid. 98, 3575 (1994).

${ }^{3}$ Y. Kanemitsu, Phys. Rev. B 48, 12357 (1993).

${ }^{4}$ H. Takagi, H. Ogawa, Y. Yamazaki, A. Ishizaki, and T. Nakagiri, Appl. Phys. Lett. 56, 2379 (1990). 
${ }^{5}$ X.-n. Liu, X.-w. Wu, X.-m. Bao, and Y.-1. He, Appl. Phys. Lett. 64, 220 (1994).

${ }^{6}$ M. Rückschloss, B. Landkammer, and S. Vepřek, Appl. Phys. Lett. 63, 1474 (1993); M. Rückschloss, O. Ambacher, and S. Vepřek, J. Lumin. 57, 1 (1993).

${ }^{7}$ R. E. Hummel, M. H. Ludwig, and S.-S. Chang, in Microcrystalline and Nanocrystalline Semiconductors, edited by L. Brus, M. Hirose, R. W. Collins, F. Koch, and C. C. Tsai, MRS Symposia Proceedings No. 358 (Materials Research Society, Pittsburgh, 1995), p. 151.

${ }^{8}$ D. A. Grützmacher, E. F. Steigmeier, H. Auderset, R. Morf, B. Delley, and R. Wessicken, in Microcrystalline and Nanocrystalline Semiconductors, Ref. 7, p. 833.

${ }^{9}$ D. J. DiMaria, J. R. Kirtley, E. J. Pakulis, D. W. Dong, T. S. Kuan, F. L. Pesavento, T. N. Theis, J. A. Cutro, and S. D. Brorson, J. Appl. Phys. 56, 401 (1984).

${ }^{10}$ P. Mutti, G. Ghislotti, S. Bertoni, L. Bonoldi, G. F. Cerofolini, and L. Meda, Appl. Phys. Lett. 66, 851 (1995).

${ }^{11}$ M. K. Lee and K. R. Peng, Appl. Phys. Lett. 62, 3159 (1993).

${ }^{12}$ Y. Kanemitsu, Phys. Rev. B 49, 16845 (1994).

${ }^{13}$ W. L. Wilson, P. F. Szajowski, and L. E. Brus, Science 262, 1242 (1993).

${ }^{14}$ Y. Kanemitsu, T. Ogawa, K. Shiraishi, and K. Takeda, Phys. Rev. B 48, 4883 (1993).

${ }^{15}$ G. W. 't Hooft, Y. A. R. R. Kessener, G. L. J. A. Rikken, and A. H. J. Venhuizen, Appl. Phys. Lett. 61, 2344 (1992).

${ }^{16}$ F. Koch, V. Petrova-Koch, and T. Muschik, J. Lumin. 57, 271 (1993).

${ }^{17}$ S. B. Zhang and A. Zunger, Appl. Phys. Lett. 63, 1399 (1993).

${ }^{18}$ P. Saeta, J. F. Federici, R. J. Fischer, B. I. Greene, L. Pfeiffer, R. C. Spitzer, and B. A. Wilson, Appl. Phys. Lett. 54, 1681 (1989).

${ }^{19}$ M. S. Hybertsen, Phys. Rev. Lett. 72, 1514 (1994).

${ }^{20}$ A. S. Amour, J. C. Sturm, Y. Lacroix, and M. L. W. Thewalt, Appl. Phys. Lett. 65, 3344 (1994).

${ }^{21}$ H. Presting, U. Menczigar, and H. Kibbel, J. Vac. Sci. Technol. B 11, 1110 (1993).

${ }^{22}$ K. Chen, X. Huang, J. Xu, and D. Feng, Appl. Phys. Lett. 61, 2069 (1992).

${ }^{23}$ X. Huang, W. Shi, K. Chen, S. Yu, and D. Feng, in Microcrystalline and Nanocrystalline Semiconductors, Ref. 7, p. 839.

${ }^{24}$ A. J. Kontkiewicz, A. M. Kontkiewicz, J. Siejka, S. Sen, G. Nowak, A. M. Hoff, P. Sakthivel, K. Ahmed, P. Mukherjee, S. Witanachchi, and J. Lagowski, Appl. Phys. Lett. 65, 1436 (1994).

${ }^{25}$ H. Tamura, M. Rückschloss, T. Wirschem, and S. Vepřek, Appl. Phys. Lett. 65, 1537 (1994).

${ }^{26}$ P. N. Saeta and A. C. Gallagher, J. Appl. Phys. 77, 4639 (1995).

${ }^{27}$ V. Petrova-Koch, T. Muschik, A. Kux, B. K. Meyer, F. Koch, and V. Lehmann, Appl. Phys. Lett. 61, 943 (1992).

${ }^{28}$ SIMOX stands for separation by implantation of oxygen. The SIMOX wafers discussed in this paper were obtained from SOITEC, Inc. This commercial material is identified to specify its characteristics. This does not imply recommendation or endorsement by NIST, nor does it imply that this is the best available material for the purpose.

${ }^{29}$ M. K. El-Ghor, S. J. Pennycook, F. Namavar, and N. H. Karam, Appl. Phys. Lett. 57, 156 (1990); A. Wittkower, M. Guerra, B. Cordts, R. Dolan, and P. Sandow, Nucl. Instrum. Methods Phys. Res. Sect. B 55, 842 (1991).

${ }^{30}$ S. K. Ghandhi, VLSI Fabrication Principles: Silicon and Gallium Arsenide (Wiley, New York, 1994).
${ }^{31}$ Y. Takahashi, T. Ishiyama, and M. Tabe, Appl. Phys. Lett. 65, 2987 (1994).

${ }^{32}$ Handbook of Optical Constants of Solids, edited by E. D. Palik (Academic, Orlando, 1985).

${ }^{33}$ M. Born and E. Wolf, Principles of Optics (Pergamon, Oxford, 1980), p. 627.

${ }^{34}$ G. E. Jellison, Jr. and F. A. Modine, Appl. Phys. Lett. 41, 180 (1982).

${ }^{35}$ W. H. Press, B. P. Flannery, S. A. Teukolsky, and W. T. Vetterling, Numerical Recipes (Cambridge University Press, Cambridge, England, 1987).

${ }^{36}$ H. V. Nguyen, Y. Lu, S. Kim, M. Wakagi, and R. W. Collins, Phys. Rev. Lett. 74, 3880 (1995).

${ }^{37}$ D. M. Tanenbaum, A. Laracuente, and A. C. Gallagher, Growth and Nucleation of Hydrogenated Amorphous Silicon on Silicon, MRS Symposia Proceedings No. 377 (Materials Research Society, Pittsburgh, 1995), p. 143; D. M. Tanenbaum, Ph.D. thesis, University of Colorado, 1995.

${ }^{38}$ Details of the deposition chamber may be found in D. M. Tanenbaum, Ph.D. thesis, University of Colorado, 1995.

${ }^{39}$ The sample was made from $p$-type $\mathrm{Si}(100), 10-20 \Omega \mathrm{cm}$, using a $\mathrm{HF}: \mathrm{H}_{2} \mathrm{SO}_{4}: \mathrm{NaNO}_{2}: \mathrm{H}_{2} \mathrm{O}$ etch described in M. T. Kelly, J. K. M. Chun, and A. B. Bocarsly, Appl. Phys. Lett. 64, 1693 (1994).

${ }^{40}$ H. I. Liu, N. I. Maluf, R. F. W. Pease, D. K. Biegelsen, N. M. Johnson, and F. A. Ponce, J. Vac. Sci. Technol. B 10, 2846 (1992); H. I. Liu, D. K. Biegelsen, N. M. Johnson, F. A. Ponce, and R. F. W. Pease, ibid. 11, 2532 (1993).

${ }^{41}$ The apparent decrease in oxidation rate may arise when a fraction $f$ of the layer has been entirely consumed. If the remainder oxidizes at an undiminished rate $r$, the average layer thickness decreases at the reduced rate $(1-f) r$.

${ }^{42}$ The room-temperature etch rate of $1.9 \mathrm{~nm} / \mathrm{h}$ is reported in P. B. Fischer, K. Dai, E. Chen, and S. Y. Chou, J. Vac. Sci. Technol. B 11, 2524 (1993).

${ }^{43}$ P. O. Hahn and M. Henzler, J. Vac. Sci. Technol. A 2, 574 (1984); J. Appl. Phys. 52, 4122 (1981).

${ }^{44}$ S. M. Goodnick, D. K. Ferry, C. W. Wilmsen, Z. Liliental, D. Fathy, and O. L. Krivanek, Phys. Rev. B 32, 8171 (1985).

${ }^{45}$ N. Sato and T. Yonehara, Appl. Phys. Lett. 65, 1924 (1994).

${ }^{46}$ C. Delerue, G. Allan, and M. Lannoo, Phys. Rev. B 48, 11 (1993).

${ }^{47}$ L. Tsybeskov, J. V. Vandyshev, and P. M. Fauchet, Phys. Rev. B 49, 7821 (1994).

${ }^{48}$ J. C. Vial, A. Bsiesy, F. Gaspard, R. Hérino, M. Ligeon, F. Muller, and R. Romestain, Phys. Rev. B 45, 14171 (1992).

${ }^{49}$ A. Bsiesy, F. Muller, M. Ligeon, F. Gaspard, R. Hérino, R. Romestain, and J. C. Vial, Phys. Rev. Lett. 71, 637 (1993).

${ }^{50}$ L. T. Canham, W. Y. Leong, M. I. J. Beale, T. I. Cox, and L. Taylor, Appl. Phys. Lett. 61, 2563 (1992).

${ }^{51}$ Large-area silicon "quantum walls" have recently been fabricated by photolithography and anisotropic etching (S. H. Zaidi, A. -S. Chu, and S. R. J. Brueck, in Microcrystalline and Nanocrystalline Semiconductors, Ref. 7, p. 957). Although passivated only by native oxide, which is known to have a higher defect density than high-temperature oxide, these layers are reported to luminesce as strongly as porous silicon, although no absolute efficiency is reported. This work suggests that similar photoluminescence intensities are possible from two-dimensional and zero-dimensional silicon structures.

${ }^{52} \mathrm{~F}$. Koch (private communication). 
${ }^{53}$ R. Kakkad, J. Smith, W. S. Lau, S. J. Fonash, and R. Kerns, J. Appl. Phys. 65, 2069 (1989); R. Kakkad, S. J. Fonash, and S. Weideman, Appl. Phys. Lett. 59, 3309 (1991).

${ }^{54}$ R. R. Razouk and B. E. Deal, J. Electrochem. Soc. 126, 1573 (1979).

${ }^{55}$ Standard commercial SIMOX is prepared from $\mathrm{Si}(001)$ substrates and the processing is optimized to minimize channeling of the $\mathrm{O}^{+}$during implantation. Channeling leads to the uneven distri- bution of $\mathrm{O}$ following implantation and variation in the oxide thickness following annealing. In principle it is possible to adjust the implantation procedure to minimize channeling effects on $\mathrm{Si}(110)$ and $\mathrm{Si}(111)$ substrates, but since such substrates are not commercially in demand, the procedure was unavailable from SOITEC.

${ }^{56}$ J. P. Proot, C. Delerue, and G. Allan, Appl. Phys. Lett. 61, 1948 (1992). 